\title{
The Challenge of Inflammatory Bowel Disease Diagnosis in Asia
}

\author{
Yue Li Jia-ming Qian \\ Division of Gastroenterology, Peking Union Medical College Hospital, Chinese Academy of Medical Sciences and \\ Peking Union Medical College, Beijing, China
}

\section{Key Words}

Asia · Diagnosis · Disease mimicking · Diversity ·

Inflammatory bowel disease

\begin{abstract}
Background: The current major clinical challenge relates to the accurate diagnosis of inflammatory bowel disease (IBD) in Asian countries with a high background prevalence of infections. Summary: The diagnosis of Crohn's disease in geographical areas where tuberculosis is endemic poses a major diagnostic challenge. Key Messages: Infections can mimic IBD and complicate the course of existing IBD. Limited availability of diagnostic modalities and health service may delay diagnosis. A multidisciplinary approach, continuous medical education, and academic activities may warrant an accurate diagnosis of IBD.

๑) 2016 S. Karger AG, Basel
\end{abstract}

\section{Introduction}

In Asia, an increasing incidence and prevalence of inflammatory bowel disease (IBD) has been witnessed. Over 1 decade, there has been a steady rise in the mean annual incidence of Crohn's disease (CD) and ulcerative colitis in one district of Seoul from 0.05 and 0.34 , respectively, to 1.34 and 3.08 per 100,000 inhabitants, respec-

\section{KARGER}

E-Mail karger@karger.com

www.karger.com/iid tively [1]. More recently, the incidences of IBD in Mainland China in population-based settings are reported to be $1.77,1.96$, and 3.14 in Daqing (Northern China), Wuhan, and Guangzhou (Southern China), respectively [24]. The incidence of IBD in Hong Kong, China, has risen threefold in the past decade. From results of the AsiaPacific Crohn's and Colitis Epidemiology Study (ACCESS), the crude annual overall incidence of IBD was 1.37 per 100,000 individuals in Asia, and China had the highest incidence among 8 countries in Asia (3.44 per 100,000 individuals) [5]. Given the increasing incidence of IBD in Asia and a high prevalence of intestinal infections in most Asian countries, as well as the absence of a diagnostic gold standard, we are facing more diagnostic and management challenges than western countries. In this review, we aimed to outline the diseases that can mimic IBD and the diversity of diagnostic quality for IBD in Asia.

\section{Diseases Mimic IBD}

\section{Intestinal Tuberculosis}

The diagnosis of CD in geographic areas where tuberculosis (TB) is common poses a real diagnostic challenge. CD and intestinal TB (ITB) are both chronic granulomatous disorders with overlapping clinical, pathologic, radiologic, and endoscopic findings. Mimics of these two diseases in clinical manifestations as well as the absence of 
Fig. 1. a Typical longitudinal ulcer in a patient with CD. b Typical transverse ulcer in a patient with ITB. c Round, single, large ulcer at the ileocecal area in a patient with BD. d Chronic schistosomal colitis confirmed by biopsy histology.
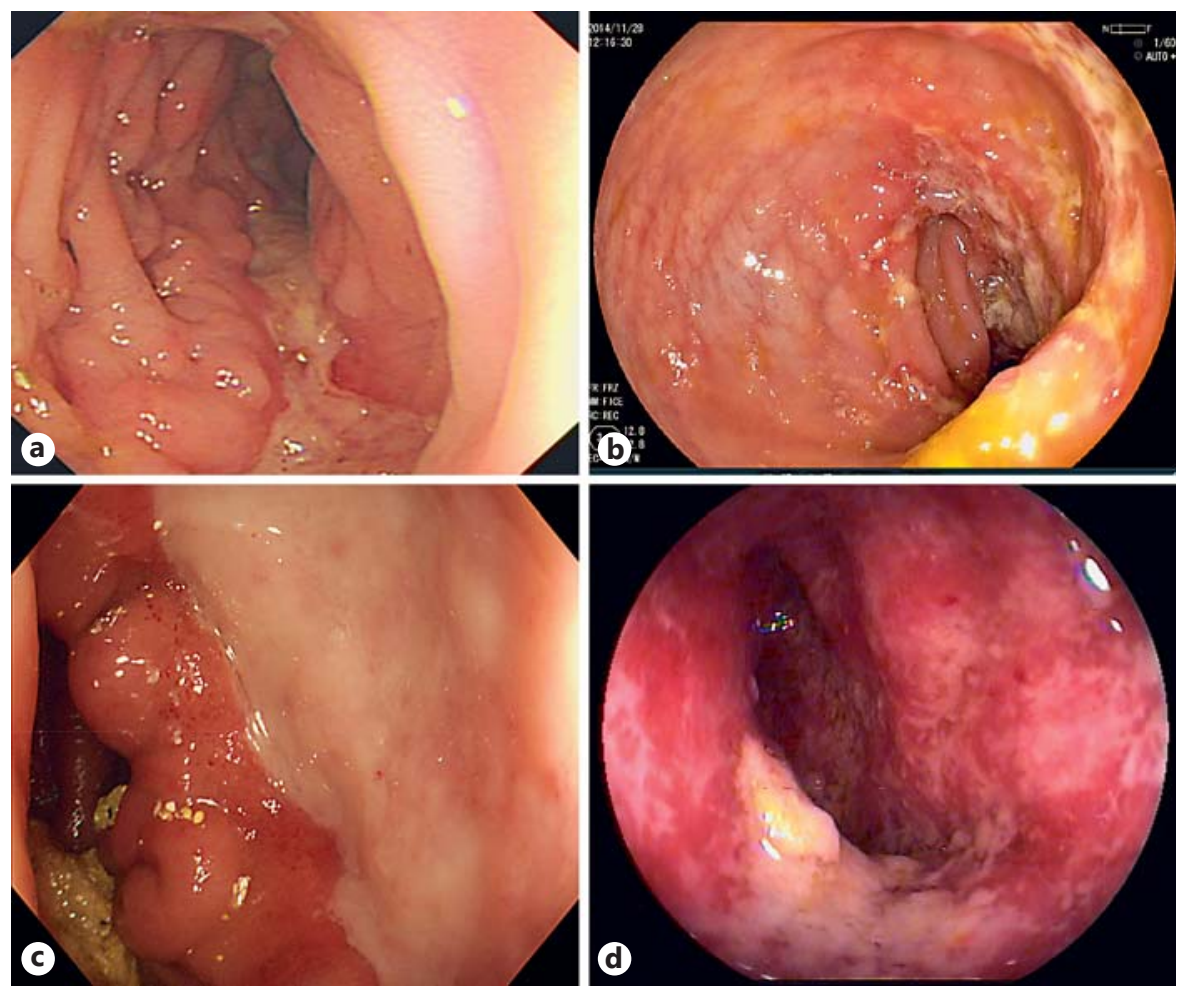

both sensitive and specific laboratory markers may explain the high misdiagnosis rates, which range from 50 to $70 \%$ [6]. Misdiagnosis and subsequent treatment may lead to unwanted consequence. To this end, many clinical researches have investigated the role of clinical presentations, colonoscopy, histopathology, interferon $\gamma$-release assay (IGRA), polymerase chain reaction (PCR)-based detection of Mycobacterium tuberculosis, and comprehensive scoring systems in discriminating between ITB and CD.

Colonoscopy might be a useful but experience-based and subjective measurement for differential diagnosis. A study from Korea in 2006 reported that the diagnosis of either ITB or CD by colonoscopy would have been made correctly in $87.5 \%$ patients $(77 / 88)$, incorrectly in $8.0 \%$ patients $(7 / 88)$, and undetermined in $4.5 \%$ patients $(4 / 88)$ [7]. A Chinese study consisting of 122 cases of ITB and 130 cases of $\mathrm{CD}$ developed a regression mathematical equation according to the endoscopic parameters as follow: rectum involvement, longitudinal ulcers, transverse ulcers, rodent-like ulcers, cobblestone sign, and fixedopen ileocecal valve (fig. 1a, b) [8]. To some extent, the colonoscopic features are poor-defined and subjective to the practitioner. In addition, patients presenting with untypical endoscopic appearance could not benefit from the results of above studies.
Endoscopic biopsy diagnosis of CD is problematic due to lack of specific microscopic features and patchy involvement. A Singapore study assessed the initial mucosal biopsy features of 25 CD, 3 ITB and 2 diverticular disease-associated colitis cases [9]. Granulomata were seen in 10 of $41 \mathrm{CD}$ biopsies, in all $5 \mathrm{~TB}$ and in no diverticular disease biopsies. Small, tight, well-defined granulomata characterized CD over large coalesced granulomata of TB. Paneth cell and pseudopyloric metaplasia was seen only in CD (2/25) [9]. Due to the low sensitivity of relatively specific features of endoscopic biopsy, it is challenging for making an accurate diagnosis entirely on the ground of biopsy histology.

To date, the role of IGRA in differentiating ITB from $\mathrm{CD}$ in Asia has been well recognized. A systematic review with a meta-analysis of the accuracy of IGRA in Asia was published in 2014 [6]. The sensitivity, specificity, positive predictive value, and negative predictive value of $\mathrm{T}$ SPOT.TB tests of 8 studies were $81,85,78$, and $87 \%$, respectively. In settings with high TB incidence such as China, where latent infection is widespread, a positive TSPOT.TB result may not necessarily discriminate active from latent TB [10]. Caution is needed in interpreting a positive T-SPOT.TB result. However, in TB endemic regions, a negative T-SPOT.TB result is very helpful to rule 
Table 1. Clinical, endoscopic, laboratory, radiological, and histological features of CD and ITB

\begin{tabular}{lll}
\hline Features & CD & ITB \\
\hline $\begin{array}{l}\text { Clinical } \\
\text { Perianal disease }\end{array}$ & Common \\
Blood in stool & Presence & Rare \\
\hline Endoscopy & & Rare \\
$\begin{array}{l}\text { Distribution } \\
\text { Ulcer appearance }\end{array}$ & $\geq 4$ segments & $<4$ segments \\
Patulous ileocecal valve & Longitudinal & Transverse \\
\hline T-SPOT.TB & Rare & Common \\
Active pulmonary TB (chest X-ray/CT scan) & Nogative & Positive (higher counts) \\
\hline $\begin{array}{l}\text { CT enterography } \\
\text { Multisegmental involvement }\end{array}$ & & Presence \\
$\begin{array}{l}\text { Sigmoid colon/rectum involvement } \\
\text { Asymmetric distribution }\end{array}$ & Common & \\
Comb sign & Common & Less common \\
Fibrofatty change & Common & Less common \\
\hline Biopsy histology & Common & Less common \\
Focal chronic inflammation & Common & Less common \\
Granuloma & & Less common \\
\hline
\end{tabular}

out ITB when distinguishing it from $\mathrm{CD}$, supported by the high negative predictive value (94.2\%) reported in a previous study of our group [11]. Intermediate or lower counts of IGRA results may be of less clinical significance and delay the diagnosis of CD.

PCR assay also helps to differentiate ITB from CD by detecting $M$. tuberculosis DNA in biopsies or fecal specimens. The reported sensitivity and specificity of the mucosal biopsy TB-PCR assay were 64.1 and $100 \%$, respectively [12]. However, false-positive or negative results may be encountered if the primer is not specific enough or the quantity of tissue available in mucosal biopsy specimens is limited. It is reported that M. tuberculosis DNA is also detected in mucosal and fecal specimens of some CD patients due to the presence of latent TB $[13,14]$. Given the confusing results from the abovementioned studies, PCR assay may have a limited diagnostic role.

Computed tomographic (CT) enterography also has a certain role in disease differentiation. Segmental involvement, comb sign, fibrofatty changes, moderate wall thickening, and asymmetric distribution were significantly more common in patients with CD than in those with ITB [15]. By combining CT enterography with colonoscopic findings, the accuracy of diagnosing either CD or ITB is increased from 66.7 to $95.2 \%$ [16]. In addition, concur-

The Challenge of IBD Diagnosis in Asia rent active pulmonary TB discovered by a CT scan can add more value to the diagnosis of ITB.

Therefore, some scoring systems have been promoted to differentiate these two diseases in a more comprehensive way $[17,18]$. To date, none of the present scoring systems is both practical and widely validated in TB endemic regions. The differentiation between $\mathrm{CD}$ and ITB in Asia is most challenging for the reasons mentioned above; a prospective, comprehensive and widely validated scoring system is imperative. The features that are valuable in the differentiation are listed in table 1.

\section{Behçet's Disease}

Behçet's disease (BD) is a chronic multisystem inflammatory disease most prevalent in Eastern Asia and along the Mediterranean basin, an area referred to as the 'Silk Road'. The diagnosis of BD mostly rests on clinical manifestations as defined in the International Study Group (ISG) criteria, which includes recurrent oral aphthae $(\geq 3$ / year) plus 2 of the following: genital aphthae, eye lesions, skin lesions, and/or positive pathergy 'skin prick' test [19]. Intestinal BD is characterized by gastrointestinal manifestations, which include, but are not limited to, chronic abdominal pain, diarrhea, gastrointestinal bleeding, mucosal ulceration, and bowel perforation. No worldwide consensus has been established for the diag-

Inflamm Intest Dis 2016;1:159-164 DOI: $10.1159 / 000448384$ 
Table 2. The clinical and endoscopic features of CD and BD

\begin{tabular}{|c|c|c|}
\hline Features & $\mathrm{CD}$ & $\mathrm{BD}$ \\
\hline \multicolumn{3}{|l|}{ Clinical } \\
\hline Abdominal pain & Usually present with intestinal obstruction & Prominent pain without signs of intestinal obstruction \\
\hline Oral ulcer & Common & $\geq 3 /$ year, painful \\
\hline Genital ulcer & No & Presence \\
\hline \multicolumn{3}{|l|}{ Endoscopic } \\
\hline Ulcer shape & Longitudinal & Round \\
\hline Distribution & Segmental & Focal, single \\
\hline Involved sites & Ileocecal & Ileocecal \\
\hline
\end{tabular}

nosis of intestinal BD. In a previous study, up to $50 \%$ of the intestinal BD patients required surgical intervention due to intestinal perforations, gastrointestinal bleeding, and fistulae formation [20].

Intestinal $\mathrm{BD}$ and $\mathrm{CD}$ commonly have a young age of onset, nonspecific gastrointestinal symptoms, similar extraintestinal manifestations, and a chronic, waxing and waning course. The mimics of these two diseases and the lack of reliable diagnostic criteria make the differentiation more difficult. A retrospective study reported that extraintestinal systemic manifestations and the characteristic colonoscopic features, such as ulcer distribution, size and type, helped to distinguish intestinal BD from CD [21]. Focal involvement, ileocecal valve deformity, solitary ulcers, large ulcers (ulcer size $>2 \mathrm{~cm}$ ), and circumferential ulcers were more common in intestinal BD patients (fig. 1c) [21]. A Korean study proposed a novel and simple diagnostic criterion on the basis of two aspects: colonoscopic findings and extraintestinal manifestations [22]. This added additional values especially in patients with ileocolonic ulcers who do not fully fulfil the diagnostic criteria of systemic BD.

It is believed that misdiagnosis of intestinal BD with $\mathrm{CD}$ usually brings less harm to patients' outcome. Deliberate differentiation of the diseases will help clinicians select appropriate immunomodultors. For instance, the first-line immunosuppressant is commonly cyclophosphamide for patients with intestinal $\mathrm{BD}$ as well as azathioprine for patients with $\mathrm{CD}$. In table 2, the clinical and endoscopic features of $\mathrm{CD}$ and $\mathrm{BD}$ are listed.

\section{Other Infectious Colitis}

Infectious colitis typically presents with a sudden symptom onset, which is also referred to as acute selflimited colitis. In developing and rural areas in Asia, in- fectious colitis remains to be one of the most common causes of diarrhea that may mimic IBD. Colitis can be caused by bacterial and parasitic infections, ileitis can result from Yersinia and Salmonella infections, and ileocolonic ulcers can be seen in amebiasis.

Symptoms of acute infection are sudden onset, early fever, and more than 6 bowel movements daily. In IBD, the symptom onset is gradual, and fever, when present, usually occurs later in the disease course. Extraintestinal symptoms and signs such as arthropathy, ocular and skin symptoms can present with acute self-limited colitis but are more common with IBD.

Stool cultures have a significant role in confirming the diagnosis of infectious colitis. For patients highly suspected with ASCL, a negative stool culture does not rule out infection due to the sensitivity of the stool culture. Sigmoidoscopy or colonoscopy with a mucosal biopsy can be very helpful. In infectious colitis, crypt architecture is normal, and mucosal inflammation is predominantly acute; there is no increase in plasma cells or lymphoid aggregates at the crypt bases. On the contrary, biopsies in IBD, even in the early course, often show crypt distortion, basilar plasmacytosis, and basal lymphoid aggregates, as well as increased acute and chronic inflammatory cells in the lamina propria [23].

Chronic schistosomal colitis can mimic ulcerative colitis in colonoscopic findings (fig. 1d) [24]. It used to be epidemic at a large scale in the regions along the Yangtze River in Southern China 50 years ago. Thanks to the effective prevention, it only occurs in a small number of people in its endemic region. An accurate diagnosis can be established by endoscopy in combination with its pathologic characteristics.

Intestinal amebiasis needs to be included in the differential diagnosis of IBD in endemic countries such as
Li/Qian 
India, China, and others. Some endoscopic and histologic features could be useful for differential diagnosis. Discrete small ulcers, $2 \mathrm{~cm}$ or less in diameter, in the cecum or rectosigmoid, were the most common finding on endoscopy in patients with amebiasis. On histology, amebic trophozoites are most frequently located within the necrotic material, mucin, proteinaceous material, and blood clot lining ulcers [25].

Infections not only mimic IBD but can also impose on and aggravate the course and outcome of IBD. Thorough screening of infections is always necessary before making the diagnosis of IBD and initiating immunosuppressive treatments on patients with IBD in Asia.

\section{Miscellaneous Diseases Mimicking IBD}

A variety of diseases on the basis of vascular etiology can mimic IBD, for example, ischemic colitis, systemic amyloidosis, and systemic vasculitis including polyarteritis nodosa, Churg-Strauss syndrome, systemic lupus erythematosus, and Henoch-Schönlein purpura. Detailed patient history and physical examination can provide clues for differential diagnosis, direct the diagnostic workup, and may expeditiously lead to an underlying cause.

Drug-induced bowel injury needs to be ruled out by a comprehensive medical history. The use of nonsteroidal anti-inflammatory drugs, proton-pump inhibitors, sodium phosphate, and mycophenolate mofetil, etc., should always raise the suspicion for drug-induced bowel injury.

\section{Diversity of Diagnostic Quality}

There is a huge diversity of health care quality in the different geographic areas in Asia owing to the economic imbalance. In certain regions, limited access of patients to health care facilities, poor physician and patient awareness of disease, and limited availability of diagnostic modalities, in particular radiologic imaging and colonoscopies, may delay diagnosis. In some countries where infections are endemic, health care providers might simply assume that abdominal symptoms are due to infections or TB without performing endoscopy. It was reported that the misdiagnosis rates between $\mathrm{CD}$ and ITB ranged from 50 to $70 \%$ [26].

To date, there are guidelines on IBD diagnosis and management in Japan, China, and Korea to help making the diagnosis accurate and standardized. Constant collaboration, academic activities, and continuous education within and between countries provide great opportunities to improve the quality of health care.

\section{Conclusion}

The incidence of IBD has increased in the past 2 decades in Asia and it is believed to continue to surge in the next decade. Given the high prevalence of infectious colitis, $\mathrm{TB}$, and $\mathrm{BD}$ in Asia, thorough differentiation is always necessary before making the diagnosis of IBD. A multidisciplinary approach, national guidelines, continuous education, as well as academic activities may help narrowing the gap of diagnosis capability among centers in different geographic areas in Asia.

\section{References}

1 Yang SK, Yun S, Kim JH, et al: Epidemiology of inflammatory bowel disease in the SongpaKangdong district, Seoul, Korea, 1986-2005: a KASID study. Inflamm Bowel Dis 2008; 14: 542-549.

2 Yang $\mathrm{H}, \mathrm{Li} \mathrm{Y}, \mathrm{Wu} \mathrm{W}$, et al: The incidence of inflammatory bowel disease in Northern China: a prospective population-based study. PLoS One 2014;9:e101296.

3 Zhao J, Ng SC, Lei Y, et al: First prospective, population-based inflammatory bowel disease incidence study in mainland of China: the emergence of 'western' disease. Inflamm Bowel Dis 2013;19:1839-1845.

4 Zeng Z, Zhu Z, Yang Y, et al: Incidence and clinical characteristics of inflammatory bowel disease in a developed region of Guangdong Province, China: a prospective populationbased study. J Gastroenterol Hepatol 2013;28: 1148-1153.

The Challenge of IBD Diagnosis in Asia
5 Ng SC, Tang W, Ching JY, et al: Incidence and phenotype of inflammatory bowel disease based on results from the Asia-Pacific Crohn's and Colitis Epidemiology Study. Gastroenterology 2013;145:158-165.

6 Ng SC, Hirai HW, Tsoi KKF, et al: Systematic review with meta-analysis: accuracy of interferon-gamma releasing assay and anti-Saccharomyces cerevisiae antibody in differentiating intestinal tuberculosis from Crohn's disease in Asians. J Gastroenterol Hepatol 2014;29:1664-1670.

7 Lee YJ, Yang SK, Byeon JS, Myung SJ, Chang HS, Hong SS, Kim KJ, Lee GH, Jung HY, Hong WS, Kim JH, Min YI, Chang SJ, Yu CS: Analysis of colonoscopic findings in the differential diagnosis between intestinal tuberculosis and Crohn's disease. Endoscopy 2006; 38:592-597.
8 Li X, Liu X, Zou Y, Ouyang C, Wu X, Zhou M, et al: Predictors of clinical and endoscopic findings in differentiating Crohn's disease from intestinal tuberculosis. Dig Dis Sci 2011; 56:188-196.

9 Kumarasinghe MP, Quek TP, Chau CY, Mustapha NR, Luman W, Ooi CJ: Endoscopic biopsy features and diagnostic challenges of adult Crohn's disease at initial presentation. Pathology 2010;42:131-137.

10 Chee CB, Barkham TM, KhinMar KW, et al: Quantitative T-cell interferon-gamma responses to Mycobacterium tuberculosis-specific antigens in active and latent tuberculosis. Eur J Clin Microbiol Infect Dis 2009;28:667-670.

11 Li Y, Zhang LF, Liu XQ, Wang L, Wang X, et al: The role of in vitro interferon $\gamma$-release assay in differentiating intestinal tuberculosis from Crohn's disease in China. J Crohns Colitis $2012 ; 6: 317-323$. 
12 Gan HT, Chen YQ, Ouyang Q, Bu H, Yang $\mathrm{XY}$ : Differentiation between intestinal tuberculosis and Crohn's disease in endoscopic biopsy specimens by polymerase chain reaction. Am J Gastroenterol 2002;97:1446-1451.

13 Pulimood AB, Peter S, Rook GWA, Donoghue HD: In situ PCR for Mycobacterium tuberculosis in endoscopic mucosal biopsy specimens of intestinal tuberculosis and Crohn disease. Am J Clin Pathol 2008;129:846-851.

14 Ramadass B, Chittaranjan S, Subramanian V, Ramakrishna BS: Fecal polymerase chain reaction for Mycobacterium tuberculosis IS6110 to distinguish Crohn's disease from intestinal tuberculosis. Indian J Gastroenterol 2010;29: 152-156.

15 Park YH, Chung WS, Lim JS, Park SJ, Cheon JH, Kim TI, Kim WH, Hong SP: Diagnostic role of computed tomographic enterography differentiating Crohn disease from intestinal tuberculosis. J Comput Assist Tomogr 2013; 37:834-839.

16 Mao R, Liao WD, He Y, Ouyang CH, Zhu ZH, Yu C, Long SH, Chen YJ, Li ZP, Wu XP, Lv $\mathrm{NH}$, Hu P, Chen M: Computed tomographic enterography adds value to colonoscopy in differentiating Crohn's disease from intestinal tuberculosis: a potential diagnostic algorithm. Endoscopy 2015;47:322-329.

17 Makharia GK, Srivastava S, Das P, Goswami P, Singh U, Tripathi M, Deo V, Aggarwal A, Tiwari RP, Sreenivas V, Gupta SD: Clinical, endoscopic, and histological differentiations between Crohn's disease and intestinal tuberculosis. Am J Gastroenterol 2010;105:642-651.

18 Huang X, Liao WD, Yu C, Tu Y, Pan XL, Chen YX, Lv NH, Zhu X: Differences in clinical features of Crohn's disease and intestinal tuberculosis. World J Gastroenterol 2015;21:36503656.

19 Criteria for diagnosis of Behçet's disease. International Study Group for Behçet's Disease. Lancet 1990;335:1078.

20 Moon CM, Cheon JH, Shin JK, Jeon SM, Bok HG, Lee JH, et al: Prediction of free bowel perforation in patients with intestinal Behçet's disease using clinical and colonoscopic findings. Dig Dis Sci 2010;55:2904-2911.

21 Li J, Li P, Bai J, Lyu H, Li Y, Yang H, Shen B, Qian JM: Discriminating potential of extraintestinal systemic manifestations and colonoscopic features in Chinese patients with intes- tinal Behçet's disease and Crohn's disease. Chin Med J (Engl) 2015;128:233-238.

22 Cheon JH, Kim ES, Shin SJ, Kim TI, Lee KM, Kim SW, Kim JS, Kim YS, Choi CH, Ye BD, Yang SK, Choi EH, Kim WH: Development and validation of novel diagnostic criteria for intestinal Behçet's disease in Korean patients with ileocolonic ulcers. Am J Gastroenterol 2009; 104:2492-2499.

23 Surawicz CM, Haggitt RC, Hussemann M, McFarland LV: Mucosal biopsy diagnosis of colitis: acute self-limited colitis and acute idiopathic inflammatory bowel disease. Gastroenterology 1994;107:755-763.

24 Cao J, Liu WJ, Xu XY, Zou XP: Endoscopic findings and clinicopathologic characteristics of colonic schistosomiasis: a report of 46 cases. World J Gastroenterol 2010;16:723-727.

25 Singh R, Balekuduru A, Simon EG, Alexander M, Pulimood A: The differentiation of amebic colitis from inflammatory bowel disease on endoscopic mucosal biopsies. Indian J Pathol Microbiol 2015;58:427-432.

26 Ma JY, Tong JL, Ran ZH: Intestinal tuberculosis and Crohn's disease: challenging differential diagnosis. J Dig Dis 2016;17:155-161. 